Research Journal of Medical Sciences 4 (3): 136-141, 2010

ISSN: $1815-9346$

(C) Medwell Journals, 2010

\title{
The Cases of Cholestatic Hepatitis in the Course of Atypical Epstein-Barr Virus Infection
}

\author{
${ }^{1}$ Ocal Sirmatel, ${ }^{3}$ Fatma Sirmatel, ${ }^{2}$ Fatma Nur Eris and ${ }^{2}$ Tekin Karsligil \\ ${ }^{1}$ Department of Radiology, ${ }^{2}$ Department of Infectious Diseases, \\ School of Medicine-Bolu, University of Abant Izzet Baysal, Turkey \\ ${ }^{3}$ Department of Microbiology and Clinical Microbiology, \\ School of Medicine-Gaziantep, University of Gaziantep, Turkey
}

\begin{abstract}
Epstein Barr Virus (EBV) infection often causes a triad of fever, sore throat and adenopathy, referred to as Infectious $\mathrm{M}$ ononucleosis. Gastrointestinal manifestations are frequently present but often unrecognized. The aim of this study is to present cases who admitted emergency service with acute cholestatic hepatitis and acute abdomen pain in the course of Ebstein-Barr Virus (EBV) infection. The five cases, two of whom were male and three female were between 14 and 51 years of age. In each of the cases, cholestatic hepatitis, mesenteric lymphadenomegaly and severe abdominal pain were observed. Also two of five patients showed thickening in the wall of the gallbladder (acalculosus hydropical gallbladder), ascites and multiple lymphadenomegaly in porta hepatis, paraaortocaval and mesenteric areas by abdominal ultrasonography. These clinical cases of acute cholestatic hepatitis resolves spontaneously in EBV infections with symptomatic treatment. Therefore these cases presented in order to draw attention to the fact that there may be different presentations in EBV infections. On the other hand, clinicians should be aware of co-occurence of acute cholestatic hepatitis during EBV infections. In such cases, radiology seriously helps to diagnosis, efficient radiological consultation and ultrasonic detection prevent drug overuse and invasive procedures.
\end{abstract}

Key words: Ebstein-Barr virus infection, acute cholestatic hepatitis, acalculous cholecytitis, abdominal pain, ultrasonic diagnosis, Turkey

\section{INTRODUCTION}

Epstein-Barr virus is a member of human pathogenic herpesviruses (Linde, 2003; Shaukat et al., 2005; Chiba et al., 2004; Popper, 1972; Jacobson et al., 1984; Edoute et al., 1998). The most frequent clinical signs are severe sore throat, palatal petechiae, cervical lymphadenopathy, splenomegaly, hepatomegaly and abdominal pain (Sainsbury et al., 1994; Mandel et al., 2005).

During EBV infection can be observe many complications such as splenic rupture, neurological findings, myocarditis and granulocytopenia. Jaundice is rare and is often attributed to autoimmune hemolysis (Chiba et al., 2004). Induced cholestatic hepatitis and mesenteric lymphadenopathy are very rare in EBV infections (Dinulos et al., 1994).

In the 33 earlier reported cases-cholecystasis without gallstones-occurred between 1966 and 2008 and had been described as very rare cases (Sung et al., 1989; Yoshie et al., 2004; Prassouli et al., 2007; Taria et al., 2008; Attilakosa et al., 2009). After 2005 more cases are reported about acalculous cholecystitis. The aim of this study is to presents 5 cases of EBV infection in which the patients had severe acute abdominal pain, mesenteric lymphadenomegaly, jaundice, acute cholestatic hepatitis and fever. Ultrasonography supported and provided very useful descriptions and evidences of cholestatic hepatitis.

\section{MATERIALS AND METHODS}

CASE 1: A 14 years old male who had complained of nausea, vomiting, sore throat, jaundice, right upper quadrant abdominal pain and high fever during last 5 days. He had been diagnosed of acute viral hepatitis. Ultrasonography (US) of the abdomen showed thickening of the gallbladder wall with a pericholecystic collection and ultrasonographical Murphy's sign was positive (Fig. 1).

Abdominal Ultrasonography (US) indicated a case of acute cholecystitis with contracted and thickening of the hydropical gallbladder wall $(6.5 \mathrm{~mm})$ without gallstones mild hepatosplenomegaly, ascites with multiple reactive-

Corresponding Author: Fatma Nur Eris, Department of Radiology, University of Abant Izzet, 


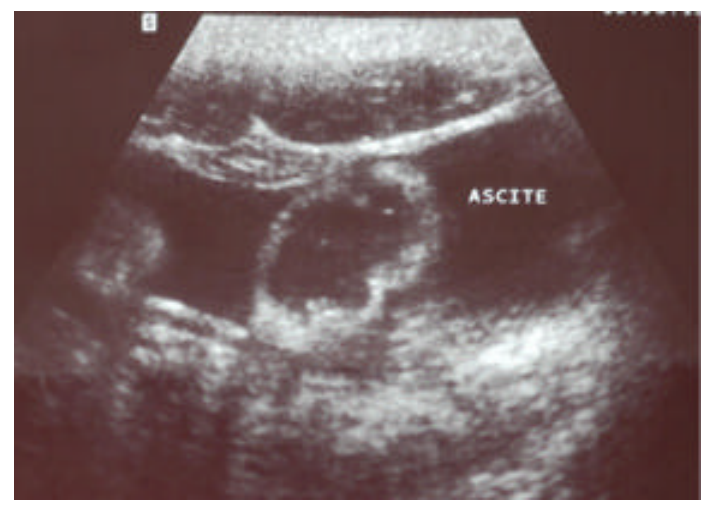

Fig. 1: Longitudinal sonogram of the gallbladder. A small sludge ball and thickened gallbladder wall $(6.5 \mathrm{~mm})$ with diffuse ascites around bowel loops in abdomen in case 1 are demonstrated

enlarged lymph nodes (between $0.5-2.5 \mathrm{~cm}$ ) in porta hepatis, paraaortocaval and mesenteric areas (Fig. 2). It was defined a positive sonographic Murphy's sign (Fig. 1). A staff doctor in emegency service directly applied to Infectious diseases and surgery consultation in case of hepatitis and acute abdomen pain consequently. In the laboratory analyses; a White Blood Cell count (WBC) of 16×109 (70\% lymphocytes), elevated hepatic enzymes (ALT: $1755 \mathrm{IU}$ [n.40], AST: $1064 \mathrm{IU}$ [n.38]), elevations in blood bilirubin (total biluribin $4.5 \mathrm{mg} \mathrm{dL}^{-1}$, direct bilirubin $38 \mathrm{mg} \mathrm{L}^{-1}$ ) and the tissue degradation enzymes (LDH: 1264 [n.490], CK-MB 127 [n.24], alkaline phosphatase $975 \mathrm{IU}$ [n.270], GGT: 267

IU [n.49]. The Enzyme Immunoassay (EIA) method (Cobas, Roche USA) detected anti-HBs (350 IU) and antiHAV I gG positivity, EBV-specific (IBL, Hamburg Germany) viral capsid antibodies (VCA-IgG: 2.253 and VCA-IgM: 2.536$)$ and positive heterophilic antibodies (Immunocard Stat Mono Test, Meridian, Diag. USA). The patient was followed up according to clinical, serological, biochemical findings and radiologically acute without gallstones cholestatic hepatitis (Fig. 1). Abnormal laboratory and radiological findings rapidly returned to their normal levels at the end of 15 days. During the follow-up of 6 months, he was in better condition without any complaint.

CASE 2: A 16 years old female patient who was admitted with abdominal pain, sore throat, jaundice and fatigue for 6 days. In the differential diagnosis of cholestatic jaundice, no evidence of acute abdomen, drug intake or autoimmune haemolysis was found. Laboratory findings suggested EBV infection. Laboratory analyses showed (WBC) of $21 \times 109$ (70\% lymphocytes). ALT: 1349, AST:
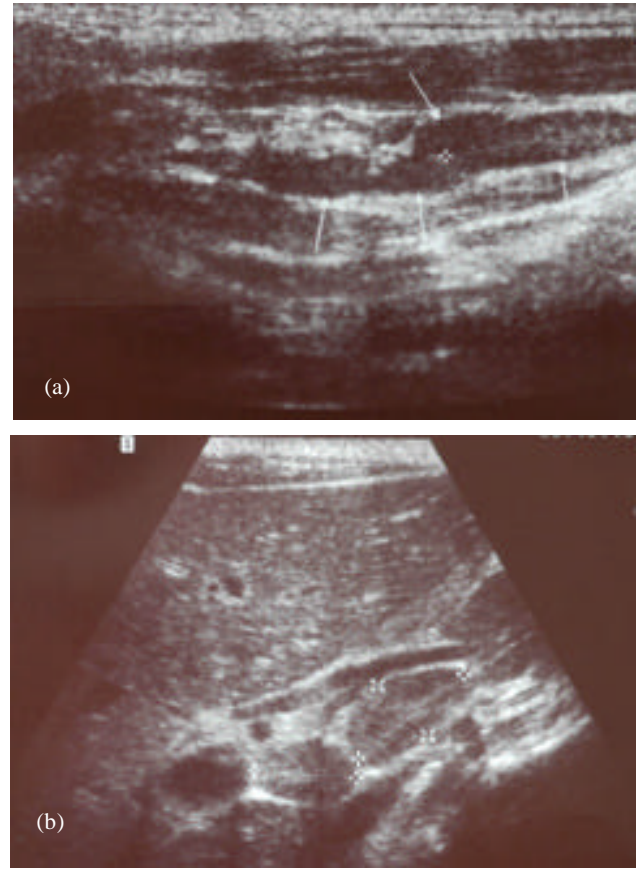

Fig. 2 a, b: Ultrasonographic image enlarged lymph nodes (between $0.5-2.5 \mathrm{~cm}$ ) in porta hepatis, paraaortocaval areas

1315, LDH: 450, CK-MB 30, alkaline phosphatase: $820 \mathrm{IU}$, GGT: $256 \mathrm{IU}$ ). Abdominal ultrasonography confirmed the presence of hepatomegaly and lymphadenomegaly in the mesenteric areas a positive sonographic Murphy's sign (Fig. 2). Using the EIA method, anti-HBs (820) and anti-HAV-IgG-positivity, EBV-specific VCA-IgG and-IgM antibodies were detected also positive. The case with acute viral hepatitis was identified as an EBV infection. Abnormal laboratory and radiological findings returned to their normal levels after 2 weeks.

CASE 3: A 26 years old female patient was admitted to the hospital with a history of acute right quadrant abdomen pain, vomiting, sore throat and oropharyngeal hyperemia, fever, chills and night sweating, general malaise, anorexia and weight loss, cholestatic jaundice and submandibular lymphadenomegaly. Costly diagnostic laboratory tests and invasive procedures were performed to rule out a malignant extrahepatic biliary obstruction. But only several tests identified the case as EBV infection. The laboratory fidings were (WBC) of $14 \times 109(70 \%$ lymphocytes). ALT: 655, AST: 564, total bilüribin $5.2 \mathrm{mg} \mathrm{dL}{ }^{-1}$, direct bilirubin $4.1 \mathrm{mg} \mathrm{L}^{-1}, \mathrm{LDH}$ : 2164 , CK-MB 227, alkaline phosphatase: 475 IU and GGT: 267 IU. EBV-specific VCA-IgG and IgM antibodies were positive. An abdominal US was performed because of 
severe abdominal pain. It showed hepatosplenomegaly and lymphadenomegaly in mesenteric areas. After symptomatic treatment, she completely recovered within 17 days.

CASE 4: A 19 years old female patient who admitted with abdominal pain, sore throat, scleral icterus and fatigue for 10 days. A broad spectrum antibiotic treatment was administered before the admission. The spleen was palpable $2 \mathrm{~cm}$ below costal margin with exudative tonsillopharyngitis and anterior cervical lymphadenopathy. In the laboratory analysis (WBC) of $17 \times 109$ (70\% lymphocytes) total bilüribin $6.9 \mathrm{mg} \mathrm{dL}^{-1}$, direct bilirubin $5.1 \mathrm{mg} \mathrm{L}^{-1}$, ALT: 879, AST: 678, LDH: 450, CK-MB 30, alkaline phosphatase: 720 IU, GGT: 236 IU. The abdominal ultrasound detected to hepatosplenomegaly, mesenteric reactive lymphadenomegaly and a thickened gall bladder wall $(3.9 \mathrm{~mm})$. EBV-specific VCA-IgG and IgM antibodies and early EBV viral antigen were all positive whereas IgG antibodies for EBV nuclear antigen were negative. Serologic studies for other hepatitis or mononucleosis agents were negative. Neither parasitic nor intracellular agents (such as Brucella or Leptospira) were identified. A monospot test (heterophile antibodies) was also positive. The patient improved in 14 days.

CASE 5: A 51 years old female patient was diagnosed of acute hepatitis 2 weeks ago when she went to a physician with complaints of sore throat, fever and fatigue. Again hepatosplenomegaly, scleral icterus, oropharyngeal hyperemia and submandibular lymphadenomegaly were defined. In the laboratory analysis, (WBC) of $19 \times 109$ (70\% 1 ymphocytes) ALT: 155, AST: 164, total biluribin $3.5 \mathrm{mg} \mathrm{dL}^{-1}$, direct bilirubin $2.6 \mathrm{mg} \mathrm{L}^{-1} \mathrm{LDH}: 1164, \mathrm{CK}-\mathrm{MB}$ 327, alkaline phosphatase: 775 IU, GGT: 467 IU were found. The abdominal US defined hepatosplenomegaly and lymphadenomegaly in mesenteric areas. Abdominal Ultrasonography (US) showed cholecystitis consisting of thickening of the wall $3.5 \mathrm{~mm}$ and hydropical gallbladder without gallstones as well as hepatosplenomegaly, ascites and in porta hepatis in paraaortic and in mesenteric areas with multiple enlarged lymph nodes. Using the EIA method, EBV-specific viral capsid antibodies (VCA-IgG: 1.977; VCA-IgM: 0.745; cut off: $0.320 ; 0.450$ ) and heterophilic antibodies were positive and the case was identified as acute EBV infection. The patient had anti$\mathrm{HBs}$ (>1000 IU), anti HAV-IgG and anti HEV-IgG positivity. After 4 weeks, cholecytitis subsided gradually as the infection regressed and the patient showed remarkable improvement like earlier 4 cases. Repeated ultrasonographic examinations showed a progressive improvement in the first ultrasonographic findings. The tests related to the liver function showed improvement and no other pathology was observed during 8-month follow-up. EBV nuclear antigen IgG antibodies became positive, confirming the diagnosis of the EBV primary infection.

\section{RESULTS AND DISCUSSION}

Patients with acute EBV infections may also develop cholestatic hepatitis with associated jaundice and hepatitis with moderate elevations in the transaminase levels. Gastrointestinal manifestations are frequently present but often unrecognized. Severe cholestasis is rare $(5 \%)$ in EBV. But physicians need to be aware of complication of cholecytitis in EBV and EBV infection should be included in the differential diagnosis of cholestasis. Acalculous cholecytitis is acute necroinflamatory disease of gallbladder with multifactorial pathogenesis. Most cases of acalculous cholecytitis are induced by infection, trauma or may be associated with a systemic vasculitis. Acute acalculous cholecytitis is a severe illness that is a complication of various other medical or surgical conditions. EBV-associated acalculous cholecytitis occured mostly in females and can affect persons of any age and almost always self-limited disease.

The 5 cases between 15 and 51 years of age and having abdominal pain with acute viral hepatitis were admitted to emergency service in the hospital. Clinical signs of EBV infection observe such as fever, pharyngitis, lympadenopathy, hepatosplenomegaly occurs in every patient during the admission. Both the 14 years old and the 51 years old who had come to the emergency unit having a sudden right-side pain, jaundice and acute cholecystitis. These 3 patients showed thickening in the wall of the hydropical gallbladder, ascites and multiple lymphadenomegaly in porta hepatis, paraaortocaval and mesenteric areas by abdominal ultrasonography.

Clinical findings (hepatomegaly and icterus) and laboratory results (elevated serum ALT, AST, alkaline phosphatase and bilirubin) were consistent with a clinical presentation of acute viral hepatitis. At the end of analyses and follow-up, the diagnosis of acute EBV infection was confirmed by the peripheral blood findings (atypical lymphocytosis) and serological markers (Specific VCA IgM- and IgG-positivity) with ELISA and a positive heterophilic antibody monospot assay.

Researchers identified 32 other cases of EBV in the literature similar to the earlier cases whereas between 1966 and 2008 English Medline (Edoute et al., 1998; Sainsbury et al., 1994; Shaukat et al., 2005; Chiba et al., 2004; Popper, 1972; Mandel et al., 2005; Dinulos et al., 1994; Sung et al., 1989; Cotton et al., 1976; Tahan et al., 2001; Massei et al., 2001; Ghosh et al., 1997; Yamada and Yamada, 2001; Maruyama et al., 2005; Yoshie et al., 2004). The characteristics of the cases and the other cases are shown in Table 1. Acute EBV infections may be seen in atypical clinical forms (Jacobson et al., 1984; Cotton et al., 
Table 1: Characteristics of cases with EBV hepatitis in literature and presented cases

\begin{tabular}{|c|c|c|}
\hline $\begin{array}{l}\text { Characteristics } \\
\text { of cases }\end{array}$ & $\begin{array}{c}\text { *Presented } \\
\text { cases:mean (range) }\end{array}$ & $\begin{array}{l}\text { ***Presented cases } \\
\text { in literature }\end{array}$ \\
\hline Median age: & 25.2 years $(14-51)$ & 20 years $(1-72)$ \\
\hline Male/female: & $2 / 3$ & $15 / 18$ \\
\hline \multicolumn{3}{|l|}{ Presenting symptoms: } \\
\hline $\begin{array}{l}\text { Cervical/general } \\
\text { lymphadenopathy }\end{array}$ & $4 / 3$ & 16 \\
\hline Paryngitis/tonsillitis & 5 & 12 \\
\hline Fever & 5 & 26 \\
\hline Jaundice & 5 & 26 \\
\hline Splenomegaly & 5 & 19 \\
\hline \multicolumn{3}{|l|}{ Liver ass ociated tests: } \\
\hline $\begin{array}{l}\text { Median total } \\
\text { biluribin (IU L }{ }^{-1} \text { ) }\end{array}$ & $5.1(3.5-6.9)$ & $12.6(2.2-47.5)$ \\
\hline $\begin{array}{l}\text { Median AST or } \\
\text { ALT }\left(\mathrm{IU} \mathrm{L}^{-1}\right)\end{array}$ & $957-1218(564-1755)$ & $179(56-2518)$ \\
\hline $\begin{array}{l}\text { Median alkaline } \\
\text { phosphatase }\left(\mathrm{IU} \mathrm{L}^{-1}\right)\end{array}$ & $753(475-975)$ & $749(31-3105)$ \\
\hline $\begin{array}{l}\text { EBV serology: } \\
\text { positive viral }\end{array}$ & 5 & 24 \\
\hline \multicolumn{3}{|l|}{ Capsid antigen IgM } \\
\hline $\begin{array}{l}\text { Mean hemoglobin } \\
\left(\mathrm{mg} \mathrm{dL}^{-1}\right) \text { : }\end{array}$ & $11.5(10-13.1)$ & 12.6 \\
\hline \multicolumn{3}{|l|}{ Ultrasound findings: } \\
\hline Hepato-splenomegaly & 5 & 6 \\
\hline Gall bladder wall thickening & 2 & 18 \\
\hline Median follow-up (range): & $\begin{array}{l}4.6 \text { months } \\
(2-8 \text { months })\end{array}$ & $\begin{array}{l}30 \text { days } \\
\text { (5-180 days) }\end{array}$ \\
\hline
\end{tabular}

*Number of 5 cases findings, **Number of 33 cases findings (Ghosh et al., 1997; Yamada and Yamada., 2001; Maruy ana et al., 2005; Yoshie et al., 2004; Prassouli et al., 2007; Iaria et al., 2008; Attilakos et al., 2009)

1976; Yoshie et al., 2004; Prassouli et al., 2007; Taria et al., 2008; Attilakosa et al., 2009). The manifestations of EBV on liver function may be a result of the stagnation of bile flow than mechanical obstruction as in classic stone disease but centrilobuler cholestasis is a consequence of the poor function of hepatocyte organelles (including mitochondria) arranged around biliary microvilli (Sainsbury et al., 1994; Popper, 1972). Jacobson et al. (1984) and Popper (1972) has reported a case of EBV infection having abdominal pain and hemolytic anemia. There is minimal hepatocellular damage associated with EBV (Ghosh et al., 1997).

During acute phase, EBV leads to systemic lymph nodes enlargement, with minimal direct hepatic invasion (Edoute et al., 1998; Mandel et al., 2005; Tahan et al., 2001). Additionally, 3 of 5 cases had acute cholecystitis without gallstones accompanied by the formation of ascites in the abdomen. Yamada and Yamada (2001) noted 6 of 39 patients with infectious mononucleosis having thickening of the walls of the gallbladder in sonography. In the study, acute cholecystitis was observed in all five patients but only three cases showed the evidence of thickening of the wall of the gallbladder.

Three of the patients had hydropical gallbladders without gallstones. In the Medline research, there were cases of gallbladder wall thickening in infectious mononucleosis (Ghosh et al., 1997; Yamada and Yamada,
2001; Maruyama et al., 2005; Yoshie et al., 2004; Prassouli et al., 2007; Iaria et al., 2008; Attilakosa et al., 2009).

In EBV infections, obstruction in biliary ducts and cholestatic hepatitis (Edoute et al., 1998) may be due to inflammation or lymphadenomegaly caused by the virus (Tahan et al., 2001; Massei et al., 2001). The edema and inflammation occurring in lymph nodes may also affect mesenteric lymph nodes which leads to severe abdominal pain (Sung et al., 1989; Cotton et al., 1976; Tahan et al., 2001; Massei et al., 2001). The severe abdominal pain in all our cases should be depend on mesenteric lymphadenomegaly. In the 3 cases, mesenteric lymphadenomegaly and cholestatic hepatitis were observed in the course of infectious mononucleosis. Therefore, ultrasonography is a useful diagnostic tool for confirming abdominal pathology and lymphadenomegaly in cases with similar symptoms, especially in early children and adult (Linde, 2003). Although, no clear consensus appears as to the sonographic criteria for acalculous cholecytitis, there are combinations of nonspesific criteria such as gallbladder wall thickening, globular distension, localized tenderness (sonographic Murphy sign), sludge, pericholesytic fluid and striated gallblader wall. A gallbladder wall thickness of $3.5 \mathrm{~mm}$ or more may be accepted to be diagnostic of acalculous cholecytitis.

Emergency cholecystectomy is the treatment of choice, once the diagnosis is established as AAC. This is because gangrene and perforation are more frequent and the outcome is worse when compared to acute calculous cholecystitis. The distention of the gallbladder $>5 \mathrm{~mm}$ in transverse diameter has also been reported. Other diagnostic ultrasonographic findings include gas, fluid and a kind of halo representation whereas halo and gas are sonolucent intramural layers and fluid has pericholecystic origin. Particularly when abdominal pain and mesenteric lymphadenitis were observed with diarrhea should be considered such as yersiniosis, salmonellosis, tuberculosis, amebiasis and malignant pathologies. The clinical constellation of fever, jaundice, right upper quadrant abdominal pain, weight loss and elevated AP levels in an older patient can suggest extrahepatic biliary obstruction. In one series, 7 of 27 patients with EBV infection had symptoms of fever, jaundice and right upper quadrant pain; 3 of them underwent detailed evaluation for biliary obstruction.

Hence, any of these etiological factors including diarrhea was not observed. The cases initially showed severe abdomimal pain and jaundice resulting from acute cholestatic hepatitis but they improved rapidly and spontaneously. In acute viral hepatitis caused by acute 
hepatotrophic viruses, the recovery period may be somewhat longer (Linde, 2003; Mandel et al., 2005). In acute EBV infections, heterophilic antibodies become positive after the 3rd week of infection (Cotton et al., 1976) resulting in the occurrence of EBV-specific VCA IgG and $\operatorname{IgM}$ positivity associated with the clinical findings (Popper, 1972; Mandel et al., 2005). In the 5 cases, no pathology was detected that may have caused mesenteric lymphadenitis in the abdomen but seropositive EBV infection was present. The symptoms in the cases including hepatitis, lymphadenitis, sore throat and the serological and biochemical results were consistent with EBV infection. These 5 cases improved merely by being given a symptomatic supportive therapy alone, without any antibiotic and steroid treatment. It is necessary for the clinicians and radiologists to be aware of the possible involvement of the gallbladder during EBV infection to avoid invasive procedures and abuse of antibiotics.

Researchers had chance to present these cases because of intimate cooperation between clinicians and radiologist. No surgical intervention was performed in the cases. The patients were treated conservatively with careful monitoring including repeated ultrasonographic examinations. In case of ultrasonographic criteria persistence and clinical deterioration on follow-up examinations, the surgical treatment would be considered. The cases were admitted to emergency in the hospital and got the chance of multidisiplinary consultations and subsequent ultrasonography. Diagnosis of EBV in emergency service requires deep consideration, especially manuel blood cells reading is important. Acute acalculous cholecytitis may occur during the course of acute EBV infection, especially in patients with cholestatic hepatitis. Researchers assume that there may be more cases of EBV associated acalculous cholecytitis than presented already.

Antibiotics are not indicated in the course of EBV-induced acalculous cholecytitis. But the patients were treated with antibiotics because the course of the disease was severe and secondary bacterial complications of gallbladder perforation were suspected. Despite antimicrobial threapy they remained febrile which also indicates EBV infection. The patients were followed carefully by serial ultrasonography which showed a large, distended gallbladder without stones. Laboratory tests showed gradual improvement but the thickness of the gallbladder wall normalized very quickly. Awareness of this unusual presentation of this common infection and early serologic diagnosis would reduce the costly diagnostic procedures often undertaken to exclude other causes of cholestasis.

All cases showed no malignant development during the 6-8 month follow-up periods. EBV induced hepatitis may be a common cause of cholestatis and should be suspected in the differential diagnosis of hyperbilirubinemia in any age group with a compatible history and physical examination. Serological analysis such as viral capsid antigen IgM or heterophile antibody (monospot test) should be ordered. As a conclusion, EBV infection should be considered in the differential diagnosis of cases with severe acute abdominal pain with cholestatis. The algorithm of the diagnosis frequently requires radiological consultation step by step. The probability of cholestatic hepatitis with mesenteric lymphadenomegaly and the clinical findings should be assessed with laboratory and radiological results. The most important point to draw attention is that in acute hepatitis patients those were admitted to hospital by severe abdominal pain and induced cholestatic hepatitis symptoms should be suspected by clinicians about infectious agents especially EBV infection. In emergency servive, the cornerstone of the consultation is whether surgical intervention is needed. At this point radiological consultation is as serious as clinical consultation.

Mild EBV-induced hepatitis is a common finding during infectious mononucleosis and usually is asymptomatic. The case series shows that more significant liver involvement may occur including jaundice, transaminase elevations and periportal inflamation and adenopathy on ultrasound examinations.

\section{CONCLUSION}

In conclusion, prompt diagnosis of EBV infection may exclude EBV mononucleosis from the list of cholestatic jaundices, thus avoiding laborious patient work-ups. Imaging studies excluded biliary obstruction. Symptoms and laboratory abnormalities resolved spontaneously. EBV infection should be included in the differential diagnosis of cholestatic hepatitis in adults.

\section{ACKNOWLEDGEMENTS}

Thanks to Dr. Resat Kervancioglu who performed radiological investigations on two patients. Written consent was obtained from the patients for the publication of these case reports.

\section{REFERENCES}

Attilakosa, A., A. Prassoulib, G. Hadjigeorgioua, E. Lagonac and S. Kitsiou-Tzelic et al., 2009. Acute acalculous cholecytitis in children with Ebstein-Barr virus infection: A role for Gilbert's syndrome? Int. J. Infect. Dis., 13: e161-e164. 
Chiba, T., S. Goto, O. Yokosuka, F. Imazeki and M. Tanaka, 2004. Fatal chronic active Epstein-Barr virus infection mimicking autoimmune hepatitis. Eur. J. Gastroenterol. Hepatol., 16: 225-228.

Cotton, W.K., J.W. McFadden and L.L. Walls, 1976. Case report: Atypical presentation of cholestatic jaundice. Postgraduate Med., 60: 259-261.

Dinulos, J., D.K. Mitchell, J. Egerton and L.K. Pickering, 1994. Hydrops of the gallbladder associated with Epstein-Barr virus infection: A case report of two cases review of the literature. Pediatric Infect. Dis. J., 13: 924-929.

Edoute, Y., Y. Baruch, J. Lachter, E. Furman, L. Basson and N. Assy, 1998. Severe cholestatic jaundice induced by Epstein-Barr virus infection in the elderly. J. Gastroenterol. Hepatol., 13: 821-824.

Ghosh, A., U.C. Ghoshal, R. Kochhar, P. Ghoshal and P.K. Banerjee, 1997. Infectious mononucleosis hepatitis: Report of two patients. Indian $\mathrm{J}$. Gastroenterol., 16: 113-114.

Iaria, C., L. Arena, G. di Maio, M.G. Fracassi, M.S. Leonardi, C. Famulari and A. Cascio, 2008. Acute acalculous cholecytitis during the course of primary Ebstein-Barr virus infection: A new case and a review of the literature. Int. J. Infect. Dis., 12: 391-395

Jacobson, I.M., D.L. Gong and R.H. Schapiro, 1984. Epstein-Barr viral hepatitis on unusual case and review of the literature. Am. J. Gastroenterol., 79: 628-632.

Linde, A., 2003. Epstein-Barr Virus. In: Manual of Clinical Microbiology, Murray, P.R., E.J. Baron, M.A. Pfaller, J.H. Jorgensen and R.H. Yolken (Eds.). American Society for Microbiology, Washington DC, pp: 1331-1340.

Mandel, G.L., J.E. Bennett and R. Dolin, 2005. Epstein-Barr virus. Princ Practice Infect. Dis., 2: 1601-1602.
Maruyama, K., H. Ushiku and Y. Kondou, 2005. Case report: Gallbladder wall thickening in children with infectious mononucleosis. J. Clin. Ultrasound, 33: $576-578$.

Massei, F., G. Palla, C. Ughi, P. Macchia and G. Maggiore, 2001. Cholestasis as a presenting feature of acute Epstein-Barr virus infection. Pediatric Infect. Dis. J., 20: 721-722.

Popper, H.H., 1972. The pathology of viral hepatitis. CMAJ, 106: 447-452.

Prassouli, A., J. Panagiotou, M. Vakaki, I. Giannatou, A. Atilakos, A. Garoufi and V. Papaevangelou, 2007. Acute acalculous cholecytitis as the initial presentation of primary Ebstein-Barr virus infection. J. Pediat. Surg., 42: e11-e13.

Sainsbury, R., P.K. Smith, L.G. Quesner, G.P. Davidson, K.F. Jureidini and D.J. Moore, 1994. Gallbladder wall thickening with infectious mononucleosis hepatitis in an immunosupressed adolescent. J. Pediatr. Gastroenterol. Nutr., 19: 123-125.

Shaukat, A., H.T. Tsai, R. Ruhterford and F.A. Anania, 2005. Ebstein-Barr virus induced hepatitis: An important cause of cholestasis. Hepatol. Res., 33: 24-26.

Sung, R.Y., R. Peck and H.G. Murray, 1989. Persitent high fever and gall-bladder wall thickening in a child with primary Epstein-Barr viral infection. J. Paediatrics Child Health, 25: 368-369.

Tahan, V., R. Ozaras, H. Uzunismail, A. Mert and F. Tabak et al., 2001. Infectious mononucleosis presenting with severe cholestatic liver disease in the elderly. J. Clin. Gastroenterol., 33: 88-89.

Yamada, K. and H. Yamada, 2001. Gallbladder wall thickening in mononucleosis syndromes. J. Clin. Ultrasound, 29: 322-325.

Yoshie, K., M. Ohta, N. Okabe, T. Komatsu and S. Umemuras, 2004. Gallbladder wall thickening with infectious mononucleosis. Abdominal Imaging, 29: 694-695. 